\title{
A PERCEPÇÃO DA VULNERABILIDADE ENTRE MULHERES COM DIAGNÓSTICO AVANÇADO DO CÂNCER DO COLO DO ÚTERO ${ }^{1}$
}

\author{
Angela Vieira Pimentel2, Marislei Sanches Panobianco ${ }^{3}$, Ana Maria de Almeida ${ }^{4}$, Iácara Santos \\ Barbosa Oliveira ${ }^{5}$
}

${ }^{1}$ Artigo extraído da dissertação - A percepção da vulnerabilidade à doença entre mulheres com diagnóstico avançado do câncer do colo do útero, apresentada ao Programa de Pós-Graduação em Enfermagem em Saúde Pública da Escola de Enfermagem de Ribeirão Preto (EERP) da Universidade de São Paulo (USP), 2010.

${ }^{2}$ Mestre em Enfermagem em Saúde Pública. Enfermeira do Hospital das Clínicas da Faculdade de Medicina de Ribeirão Preto da USP. São Paulo, Brasil. E-mail: angelavpimentel@yahoo.com.br

${ }^{3}$ Doutora em Enfermagem em Saúde Pública. Professora Doutora do Departamento de Enfermagem Materno-Infantil e Saúde Pública da EERP/USP. São Paulo, Brasil. E-mail: marislei@eerp.usp.br

${ }^{4}$ Doutora em Enfermagem em Saúde Pública. Professora Associado do Departamento de Enfermagem Materno-Infantil e Saúde Pública da EERP/USP. São Paulo, Brasil. E-mail: amalmeida@eerp.usp.br

${ }^{5}$ Mestre em Enfermagem em Saúde Pública. Enfermeira da Estratégia Saúde da Família de Passos-MG. Minas Gerais, Brasil. E-mail: iacaraoliveira@usp.br

RESUMO: Estudo descritivo, de abordagem qualitativa, que objetivou compreender a percepção da vulnerabilidade à doença, entre mulheres com diagnóstico avançado do câncer do colo do útero, tendo como referencial, a vulnerabilidade. Participaram doze mulheres que estavam em atendimento ambulatorial hospitalar, para tratamento do câncer cérvico-uterino avançado. Os dados foram coletados por entrevistas e analisados por Análise de Conteúdo. Dos resultados emergiram duas categorias temáticas: Percebendo-se vulnerável na descoberta da doença e Percebendo-se vulnerável na realização do tratamento. Fatores relacionados à cliente, profissionais, serviços, entre outros, tornaram a mulher suscetível aos problemas e danos de saúde, relativos ao câncer cérvico-uterino, exacerbando sua vulnerabilidade à doença. É necessário superar deficiências no modelo de assistência e humanização do atendimento, no grau de compromisso e qualidade das instituições, dos recursos, gerenciamento e monitoramento dos programas de prevenção e detecção do câncer do colo do útero, nos diferentes níveis de atenção.

DESCRITORES: Câncer do colo do útero. Vulnerabilidade. Prevenção de doenças. Diagnóstico tardio. Enfermagem.

\section{PERCEIVING VULNERABILITY AMONG WOMEN WITH ADVANCED DIAGNOSIS OF UTERINE CERVICAL CANCER}

\begin{abstract}
This descriptive qualitative study aimed to comprehend perceptions of vulnerability to disease among women diagnosed with advanced cervical cancer. Twelve women who were receiving outpatient hospital care in treating advanced cervical cancer participated in the study. Data was collected through interviews and analyzed via content analysis. The results highlight two themes: Perceiving oneself as vulnerable in discovering the disease; and Perceiving oneself vulnerable in carrying out treatment. Factors related to the client, health care professionals, and services rendered, among others have made women susceptible to health problems and damage relating to cervical cancer, exacerbating their vulnerability to the disease. It is necessary to overcome deficiencies in care model and the humanization of treatment, the degree of commitment, as well as the quality of institutions, resources, management and monitoring programs towards preventing and detecting cervical cancer within different levels of care.
\end{abstract}

DESCRIPTORS: Uterine cervical neoplasms. Vulnerability. Disease prevention. Delayed diagnosis. Nursing.

\section{LA PERCEPCIÓN DE LA VULNERABILIDAD ENTRE MUJERES CON DIAGNÓSTICO DE CÁNCER CERVICAL AVANZADO}

\begin{abstract}
RESUMEN: Este estudio descriptivo y cualitativo tuvo como objetivo comprender la percepción de la vulnerabilidad a la enfermedad, entre las mujeres diagnosticadas con cáncer cervical avanzado, tomando como referencia la vulnerabilidad. Participaron doce mujeres que recibían atención ambulatoria hospitalaria para el tratamiento del cáncer cervico uterino avanzado. La recolección de los datos se hizo a través de entrevistas y se analizaron mediante el análisis de contenido. Los resultados destacan dos temas: Percepción de su vulnerabilidad al descubrir la enfermedad, y percepción de su vulnerabilidad al realizar el tratamiento. Factores relacionados con el cliente, los servicios, los profesionales, entre otros, han hecho que las mujeres sean susceptibles a los problemas de salud y daños relacionados con el cáncer cervico uterino, lo que agrava su vulnerabilidad a las enfermedades. Es necesario superar las deficiencias en el modelo de tratamiento para el cuidado y la integridad personal, el grado de compromiso y calidad de las instituciones, recursos, programas de gestión y control para prevenir y detectar el cáncer del cuello uterino, en los diferentes niveles de atención.
\end{abstract}

DESCRIPTORES: Neoplasias del cuello uterino. Vulnerabilidad. Prevención de enfermedades. Diagnóstico tardio. Enfermería.

Texto Contexto Enferm, Florianópolis, 2011 Abr-Jun; 20(2): 255-62. 


\section{INTRODUÇÃO}

O câncer do colo do útero é o segundo mais comum entre as mulheres no mundo, sendo responsável, anualmente, por cerca de 500 mil casos novos e, aproximadamente, 230 mil mortes. O número de casos novos deste câncer estimados para o Brasil em 2010 é de 18.430. ${ }^{1}$ Na América Latina e Caribe é a maior causa de mortes por câncer entre mulheres. ${ }^{2}$

Esta doença se inicia a partir de uma lesão préinvasiva curável em até $100 \%$ dos casos que, normalmente, progride de forma lenta por 10 a 20 anos até atingir o estádio invasor, etapa em que a cura se torna mais difícil, quando não impossível. ${ }^{3}$

O Papiloma Vírus Humano (HPV) cuja transmissão ocorre, preferencialmente, pelo contato sexual, ${ }^{4}$ está presente em quase $100 \%$ dos casos de câncer do colo do útero, porém, para o desenvolvimento, manutenção e progressão das lesões pré-invasivas, é necessária a sua associação com os outros fatores de risco, que são o tabagismo, multiplicidade de parceiros sexuais, uso de contraceptivos orais, multiparidade, baixa ingestão de vitaminas, iniciação sexual precoce e coinfecção por agentes infecciosos, como o Vírus da Imunodeficiência Humana (HIV). . $^{5-6}$

Em nosso país, a abordagem mais efetiva para o controle deste câncer continua sendo a detecção precoce por rastreamento pelo exame de Papanicolaou, ${ }^{7}$ que é simples, de baixo custo e oferecido por toda a rede pública de saúde.

No entanto, os sinais e sintomas do câncer do colo do útero aparecem tardiamente, o que leva muitas mulheres a procurar o médico somente quando a doença já está em estádio avançado, diminuindo as chances de um tratamento menos invasivo, econsequentemente, de cura, pois o prognóstico piora com o avanço da doença. ${ }^{8} \mathrm{O}$ diagnóstico tardio, por sua vez, provoca, além de danos físicos, problemas emocionais e psicossociais, envolvendo a mulher e os que lhe são mais próximos.

Ainda é frequente que, por diversos motivos, como medo do diagnóstico, vergonha, desconhecimento da importância ou por achar o exame "embaraçoso" ou desnecessário, as mulheres deixem de realizar o exame preventivo ou o realizam, mas não voltam para saber o resultado, ou ainda, não o realizam com a periodicidade devida. ${ }^{9-11}$

Em alguns momentos, o profissional da saúde negligencia o atendimento, nem sempre realizando a coleta do material cervical para ser examinado. Ainda há falhas estruturais no processo da determinação do diagnóstico e no seguimento das mulheres com exames alterados.

Esse cenário nos mostra que fatores relacionados à cliente, aos profissionais, aos serviços, entre outros, tornam a mulher vulnerável ao câncer cérvico-uterino. Nas pesquisas em saúde, os termos "vulnerabilidade" e "vulnerável" são comumente empregados para designar suscetibilidade das pessoas a problemas e danos de saúde. ${ }^{12}$

Neste estudo buscamos compreender como tais problemas vêm se inserindo no cotidiano da mulher portadora do câncer do colo do útero.

Optamos por trabalhar com mulheres com a doença em estádio avançado, por acreditar que as suas histórias e experiências pudessem nos ajudar a responder as questões: como, porque e em que nível a vulnerabilidade à doença se apresenta a muitas mulheres, e consequentemente, visamos contribuir para reverter o quadro que descreve o cenário nacional do câncer do colo do útero. Assim, o objetivo deste estudo foi compreender a percepção da vulnerabilidade à doença, entre mulheres com diagnóstico avançado do câncer do colo do útero.

\section{REFERENCIAL TEÓRICO}

O termo vulnerabilidade significa "caráter ou qualidade de vulnerável", e vulnerável vem do latim vulnerabile: suscetível de ser ferido, ofendido ou tocado. É um termo que faz parte do vocabulário acadêmico, como também da sociedade civil, sendo usado sobre vários pontos de vista e significados. ${ }^{13}$

A vulnerabilidade engloba aspectos desde a suscetibilidade orgânica até a forma de estruturação dos programas de saúde, passando por aspectos comportamentais, culturais, econômicos e políticos. O conceito de vulnerabilidade considera, portanto, a chance de exposição das pessoas ao adoecimento como resultante de um conjunto de aspectos individuais, coletivos e contextuais, que aumentam a suscetibilidade ao adoecimento e acarretam, ao mesmo tempo, disponibilidade de recursos para se proteger de ambos. Por isso, a vulnerabilidade está articulada em três eixos interligados: o componente biológico/individual, o social e o programático/institucional. Consequentemente, qualquer mudança em um dos eixos levará consequências aos demais. ${ }^{13}$

O significado do termo vulnerabilidade refere-se, dessa forma, à chance de exposição das pessoas ao adoecimento, como resultante de um conjunto de aspectos que ainda que se refiram 
imediatamente ao indivíduo, recolocam-no na perspectiva da dupla-face, ou seja, o indivíduo e sua relação com o coletivo. Explicando melhor, o indivíduo não prescinde do coletivo, há relação intrínseca entre os mesmos. ${ }^{14}$

A vulnerabilidade individual está relacionada "ao comportamento pessoal e refere-se ao grau e à qualidade da informação de que os indivíduos dispõem sobre o problema; à capacidade de elaborar essas informações e incorporá-las aos seus repertórios cotidianos. Refere-se ainda ao interesse e às possibilidades efetivas de transformar essas preocupações em práticas protegidas e protetoras". ${ }^{13: 123}$

Já a vulnerabilidade social relaciona-se à “obtenção de informações, à possibilidade de metabolizá-las e ao poder de incorporá-las a mudanças práticas; depende do acesso aos meios de comunicação, escolarização, assim como a disponibilidade de recursos materiais, poder de influenciar decisões políticas, possibilidades de enfrentar barreiras culturais, estar livre de coerções violentas ou poder defender-se delas" ${ }^{\text {13:123 }}$

Assim, as condições culturais, econômicas e políticas precisam ser consideradas, quando se deseja compreender as razões pelas quais as pessoas pensam, fazem e querem coisas que as expõem a um agravo de longa duração ou a eventos não condizentes com a qualidade de vida. ${ }^{15}$

A vulnerabilidade programática está relacionada com os elementos de organização dos serviços; refere-se aos recursos sociais de que as pessoas necessitam para não se exporem aos agravos e se protegerem de seus danos. Do plano programático fazem parte o acesso e a forma de organização dos serviços de saúde, assim como os programas voltados à prevenção, à assistência e ao controle. ${ }^{13}$

Neste estudo, para responder as questões sobre como, porque e em que nível a vulnerabilidade à doença se apresentou às mulheres com diagnóstico avançado do câncer do colo do útero, utilizamos o modelo de vulnerabilidade que desconsidera a responsabilização única do indivíduo pela falha na prevenção às doenças, transportando-a para o âmbito coletivo. ${ }^{13}$

\section{METODOLOGIA}

Estudo descritivo e exploratório, com abordagem qualitativa, empregando a Análise de Conteúdo, ${ }^{16}$ na modalidade temática. É um estudo de campo, que utilizou entrevistas para buscar informações, entre mulheres com câncer cérvicouterino, em estádio avançado, sobre sua percepção quanto à vulnerabilidade à doença.

A coleta de dados foi realizada nos meses de abril e maio de 2009. Foram entrevistadas 12 mulheres com diagnóstico de câncer do colo do útero, em estádios IIB, III e IV, que se encontravam em tratamento em um hospital universitário do interior do estado de São Paulo, que aceitaram participar das entrevistas da pesquisa e estavam em condições clínicas que lhes permitiam falar das suas experiências, com enfoque no diagnóstico e tratamento. Por se tratar de pesquisa qualitativa, o número de participantes foi definido a partir do momento em que houve saturação de dados, ou seja, à medida que foram surgindo unidades de significação e repetição dos conteúdos, completouse a coleta. A técnica de coleta de dados foi a entrevista individual, realizada em sala de atendimento privativo, mediante uso de um formulário semiestruturado, para a caracterização dos sujeitos, seguida da entrevista com questões abertas relacionadas à vivência da mulher sobre o diagnóstico e tratamento do câncer do colo do útero.

O conteúdo das entrevistas foi transcrito e exaustivamente estudado. Considerando as motivações, as similaridades e as divergências apresentadas de forma descritiva com base nos depoimentos das mulheres. Foi possível compreender a percepção dos primeiros sintomas até o tratamento, assim como a percepção das causas de um diagnóstico tardio.

O projeto foi aprovado pelo Comitê de Ética em Pesquisa do Hospital das Clínicas da Faculdade de Medicina de Ribeirão Preto da Universidade de São Paulo, parecer n 921/2009, conforme determina a resolução 196/96 do CNS/MS. ${ }^{17}$

\section{RESULTADOS E DISCUSSÃO}

Foram entrevistadas 12 mulheres na faixa etária entre 39 e 75 anos, sendo que oito delas tinham entre 41 e 60 anos; sete apresentavam ensino fundamental incompleto como grau de instrução, e seis eram casadas. Quanto ao estádio da doença (câncer do colo do útero), sete mulheres encontravam-se em estádio IIB, três em estádio IIIB e duas em estádio IVA. As participantes tiveram o início da atividade sexual na faixa etária de 16 a 25 anos (nove) e nove mulheres relataram ter ficado grávidas de uma a cinco vezes.

A análise das informações emitidas pelos sujeitos evidenciou duas categorias temáticas: 
Percebendo-se vulnerável na descoberta da doença e Percebendo-se vulnerável na realização do tratamento.

\section{Percebendo-se vulnerável na descoberta da doença}

Durante as entrevistas, as mulheres revelaram que procuravam o serviço de saúde somente na presença de algum sinal ou sintoma. O sinal referido pela maioria era o sangramento vaginal, que incomodava por ser de grande intensidade e, muitas vezes, atrapalhava ou até mesmo impossibilitava a relação sexual. Elas sentiam que o que estava acontecendo com elas não estava dentro dos padrões normais, que sua saúde estava sendo ameaçada. A sensação de ameaça à sua saúde impunha às mulheres a percepção da sua vulnerabilidade, mesmo sem que elas soubessem, exatamente, o que estava acontecendo com elas e qual seria o desfecho a partir de então.

Começou com um pequeno sangramento, na hora de ter relação sexual, aí eu vi que não estava normal. Ficou uns três meses, quando tinha relação sangrava, aí foi aumentando, aumentando, aumentando. Aí eu resolvi procurar o médico (E1).

Começou com hemorragia, por isso que eu procurei o médico. Sangrando muito, era muito. O sangramento era muito, eu vi que não era normal, aí eu fui, o ginecologista marcou o Papanicolaou. E no exame detectou, encontrou o tumor no colo (E5).

[...] as hemorragias que eu tive diariamente, quase precisei tomar sangue. Fiquei até internada sete dias. Perdendo só sangue, sangue, sangue (E8).

Outras mulheres, além do sangramento vaginal em excesso, sem controle, referiram já apresentar sintomas mais severos, como as lesões de bexiga, que são características do câncer do colo, em estádios bastante avançados.

Tive sangramento e depois a lesão na bexiga, que a urina descia direto. Quando tive sangramento, procurei o médico (E7).

Como nas etapas iniciais do câncer cervical não há sintomas, isso pode fazer com que a pessoa não suspeite da doença e retarde a procura ao médico. No entanto, não só as mulheres com câncer invasivo, mas também aquelas com neoplasia intraepitelial cervical, somente procuram atendimento médico quando apresentam alguma queixa. ${ }^{18}$ Corroborando essas observações, outro estudo acrescenta que mulheres só buscam a prevenção do câncer do colo uterino em momentos críticos, quando o corpo manifesta que algo está errado. ${ }^{19}$
Em relação aos componentes individual e social da vulnerabilidade, ${ }^{13}$ essas falas podem revelar o grau e a qualidade da informação de que os indivíduos dispõem sobre o problema de saúde por que passam, nesse caso, o câncer do colo do útero. As mulheres podem estar retardando a procura pelo serviço médico por não terem obtido adequadamente informações sobre a importância da realização de exames preventivos, ou ainda, se as receberam, tiveram dificuldade para elaborá-las e incorporá-las no seu cotidiano de preocupações.

Há uma descontinuidade das ações desempenhadas na assistência à saúde da mulher, como educação em saúde, que se mostrou deficiente, visto que as mulheres pesquisadas por eles não tinham conhecimentos concretos acerca da prevenção do câncer do colo uterino. ${ }^{19}$

Nessa direção, questionamos as participantes desta pesquisa sobre a realização periódica do Papanicolaou, antes da descoberta da doença, ao que a maioria respondeu negativamente, conforme observamos nos trechos a seguir:

[...] não, nunca tinha feito. Eu estava trabalhando. Trabalhava na roça. Eu não tinha dor, não tinha nada. Porque eu não gostava. Tinha medo de ir ao médico (E4).

[...] nunca na minha vida. De primeiro, a gente tinha vergonha. Nossa senhora, era uma morte pra mim. Nunca fui. É a pior viagem, a gente tem que ir (E6).

[...] eu nunca tinha feito. Porque o brasileiro sempre é folgado. Sempre deixa pra depois. E depois sofre as consequências (E7).

[...] primeira vez. Nunca. Por relaxo [risos]. Verdade. Nunca fui, primeira vez (E8).

[...] não. A gente ficava trabalhando e não dava nem tempo da gente $i r$, de saber. Sabe, a patroa não dei$x a$, não gosta que sai, e fui deixando, e os anos passando, passando, e a gente sofrendo (E9).

As falas revelam que algumas das participantes deste estudo nunca haviam realizado um exame de Papanicolaou, e por motivos diversos, entre eles a vergonha. Provavelmente isso aconteça pelo fato de elas terem de se expor, pela sensação de impotência, desproteção e perda do domínio sobre o próprio corpo que a coleta da colpocitologia pode ocasionar. A exposição das partes íntimas e a posição ginecológica, exigidas para a realização do exame, o toque ginecológico, a introdução do espéculo, da espátula e da escova para coleta via vaginal estão entre os fatores que podem esquivar a mulher da coleta. 
A falta de tempo também foi citada pelas entrevistadas como motivo para a não realização do exame, gerada principalmente pelo trabalho fora de casa.

A esse respeito, estudo com 120 mulheres identificou que os principais motivos de elas se recusarem a realizar o exame de Papanicolau foram: $42 \%$ vergonha e medo, $37,5 \%$ medo do resultado, $33,3 \%$ dificuldade na marcação da consulta e 29,2\% não sabem de sua importância. ${ }^{11}$ Dados semelhantes foram observados em estudo com 267 mulheres, das quais $85,0 \%$ das entrevistadas afirmaram ter realizado o procedimento alguma vez ao longo da vida e $15,0 \%$ nunca o fizeram, por diversos motivos, dentre eles: descuido $(22,1 \%)$, não solicitação do médico $(7,4 \%)$, sentir vergonha $(6,3 \%)$, exame incomoda $(3,2 \%)$ e falta de tempo $(2,1 \%){ }^{20}$

Quando relacionamos as falas das mulheres aos componentes dos eixos de interligação utilizados para as análises de vulnerabilidade, ou seja, quando recorremos a esses componentes para analisar a forma como as mulheres lidavam com o fato de apresentar uma doença como o câncer do colo do útero, e suas atitudes perante isso, identificamos o componente individual, quando relatam a não realização do Papanicolaou antes de apresentarem a doença, por vergonha, "relaxo". Demonstraram, de certa forma, que a qualidade e o grau de informação sobre o seu problema de saúde, a elaboração dessa informação e sua aplicação prática não estavam adequados.

Isso também ocorre quando elas referem que tinham medo de ir ao médico e que não procuravam o serviço de saúde porque não tinham sintomas, ou por falta de tempo. $O$ fato de não procurarem serviço médico pelos motivos citados parece denotar que essas mulheres não tinham conhecimento da importância e necessidade da realização periódica do exame preventivo e do risco de adquirirem uma doença como o câncer do colo do útero, ou ainda por desconhecerem as consequências da doença.

Por outro lado, quando realizavam o exame, não o faziam com a periodicidade recomendada pelo Ministério da Saúde. As mulheres não buscavam o serviço de saúde ou quando o faziam, em consulta ginecológica, o profissional de saúde não realizava a coleta de citologia ou não informava acerca dos procedimentos que estavam sendo realizados. Vale destacar que algumas mulheres podem não distinguir apropriadamente entre o exame ginecológico e a coleta de material para exame de Papanicolaou.
[...] ele colheu o Papanicolaou pela primeira vez. Eu fiquei uns três anos sem ir ao ginecologista. Foi a primeira vez que eu fiz e descobri a doença (E1).

[...] já fazia tempo que eu não fazia, depois que eu tive meu último [filho], eu não fui mais; ele está com quatro aninhos. Às vezes completava um ano, eu ia lá e fazia. Às vezes eu ficava de dois anos, estava assim. Aíeu só trabalhava. Como não sentia nada, achava que não tinha precisão. Eé uma grande bobeira da gente. $O$ último tinha dado normal (E3).

[...] eu fazia de ano, depois eu fiz e nem resultado eu tive. Ainda eu perguntei lá no posto e me disseram que então não tinha dado nada. Ai, eu sentia dor no ânus, e eu consultava lá e o médico me falava que era hemorroida. Tinha sangramento. Eu falava pro médico e ele falava que era menopausa (E10).

[...] eu tinha feito um ano antes. Ele tinha me dito que tinha dado uma infecção no exame de Papanicolaou. Ele não passou nada. Em julho de 2008 começou o sangramento. Em 2009 que eu coletei o exame, em $2008 \mathrm{eu}$ não fiz porque eles diziam pra mim que não dava pra fazer coletagem nenhuma. Me consultavam e passavam remédio. Ele só mesmo me pediu os exames quando eu cheguei e falei pra ele. 'não agüento mais, não suporto, vê o que o senhor faz.'. Aí que ele coletou (E12).

Outro estudo, em que foram entrevistadas 138 mulheres, sendo 90 com diagnóstico histológico de Neoplasia Intraepitelial Cervical (NIC) de alto grau e 48 mulheres com câncer invasivo do colo uterino, identificou respostas semelhantes a estas. Entre aquelas com câncer invasivo, 63\% tinham conhecimento inadequado do exame de Papanicolaou, $81 \%$ tinham atitude inadequada em relação à necessidade de fazê-lo e $56 \%$ praticavamno de forma inadequada. Neste mesmo estudo, $60 \%$ de todas as mulheres relataram que os médicos não as examinavam durante a consulta. ${ }^{18}$

Nos relatos apresentados anteriormente identificamos componentes da vulnerabilidade programática que aponta a fragilidade da mulher diante das atitudes do profissional da saúde, em relação à prevenção e controle do câncer cérvicouterino, assim como revela deficiências no modelo de assistência, na humanização do atendimento.

As mulheres ficam à deriva de informações sobre os procedimentos que estão ou não estão sendo realizados com elas. Muitas vezes não tem conhecimento sobre seus direitos; não tem coragem de perguntar ao profissional da saúde, que lhes presta atendimento, detalhes sobre a consulta ou sobre os materiais coletados para exames, entre outros. Isso fica evidente quando elas referem que compareciam no serviço de saúde para atendimen- 
to, porém não era realizado o Papanicolaou. Em alguns casos, iam buscar o resultado do exame e este não era encontrado.

Outro dado que nos chama atenção em relação à vulnerabilidade das mulheres ao câncer do colo do útero diz respeito ao fato de que aquelas que não realizavam periodicamente o exame preventivo, não o faziam há, no máximo, cinco anos e todas apresentavam a doença em estádio avançado. Sabe-se que o câncer do colo do útero tem uma evolução lenta e que o tempo entre a lesão inicial e a fase clinicamente diagnosticável é estimado em 10 a 20 anos. ${ }^{21}$ Com base nas narrativas das mulheres acerca da periodicidade com que realizavam o exame de Papanicolaou podemos questionar como as mulheres do presente estudo puderam ser diagnosticadas tão tardiamente.

Buscar resposta a essa pergunta poderia esclarecer questões importantes acerca da efetividade do programa de controle do câncer cérvicouterino, na rede pública de saúde revelando entraves no programa que devem ser enfrentados, visando oferecer uma assistência de melhor qualidade à mulher, em relação à prevenção e controle do câncer do colo do útero.

Diante do exposto, identificamos a importância de abordar a vulnerabilidade como "a resultante de um conjunto de aspectos não apenas individuais, mas também coletivos, contextuais"13:123 pois os fatores citados acima não dizem respeito somente à mulher, e sim aos programas de prevenção e ao treinamento adequado dos profissionais, e até mesmo à gerência e monitoramento dos programas.

E mais, além de todas as consequências físicas da doença, as mulheres que apresentam o câncer em estádio avançado enfrentam também problemas emocionais e psicossociais, que podem ter sua origem a partir do diagnóstico do câncer.

O diagnóstico do câncer do colo do útero na vida das mulheres acarreta efeitos traumáticos, para além da própria enfermidade. Elas se deparam com o temor de ter uma doença sem cura, permeada por sofrimentos e estigmas, assim como a iminência da perda de um órgão que tem um valor simbólico e repleto de representações.

A partir do diagnóstico confirmado, as mulheres se deparam com suas vidas tomando um rumo diferente do que poderiam imaginar, porque o câncer pode acarretar alterações significativas nas diversas esferas da vida como trabalho, família e lazer. Dessa forma, acaba trazendo implicações em seu cotidiano e nas relações com as pessoas do seu contexto social. Vislumbrar o futuro passa a ser muito doloroso, já que os tratamentos propostos implicam em possível mutilação, náuseas, vômitos, alopecia, além de alterações sexuais.

Após a descoberta da doença, a próxima etapa é o tratamento, que muitas vezes é longo, mutilador, causa mal-estar, traz custos econômicos, entre outros.

As mulheres diagnosticadas com a doença aderem aos tratamentos propostos, preocupamse mais com a recuperação e têm esperança da cura, mesmo estando com a doença em estádio avançado.

\section{Percebendo-se vulnerável na realização do tratamento}

O medo de ter uma doença estigmatizante como o câncer, e ainda, o câncer do colo do útero, que atinge um órgão tão significativo do corpo feminino, pode ter sido um dos motivos do retardo da busca por um serviço médico a tempo de descobrir a doença em estádios iniciais, quando o tratamento poderia ter sido menos agressivo e as possibilidades de cura, maiores.

Já no momento em que vivenciam o tratamento, as mulheres convivem com uma nova realidade. Talvez não tenham ainda a percepção de todas as consequências que a doença em estádio avançado provoca, porém, sofrem com o tratamento que, por sua vez, mostra-se agressivo, mutilador, demorado. Vivem com a incerteza de um futuro promissor.

Pudemos depreender de suas falas que quando se deparam com a doença, a vulnerabilidade à maior gravidade já é percebida por elas, pois buscam seguir à risca as orientações acerca do tratamento e se utilizam, até mesmo, de outras alternativas, como aquelas contidas nas crenças populares, que poderiam auxiliar na sua recuperação.

Foi somente a radioterapia. Eu tomei muitos remédios pra dor. Fazia repouso, até hoje eu faço repouso. Nossa, eu comia que nem posso ver beterraba na minha frente [risos]. Beterraba e figado. E tomei remédio também. Bastante remédio (E8).

[...] eu tomava mais água de coco, tomava sopa, tomava bastante suco, o de limão que eu tomava só. Aí eu comecei a tomar o leite de soja também (E3).

[...] eu estava com anemia e tomei suco de jenipapo (E7).

[...] a mulher também teve câncer, aí ela falou pra mim bater laranja com maçã e beterraba, e é o que 
eu estou tomando todo dia de manhã. Estou fazendo repouso (E12).

Assim, além de seguirem corretamente a terapêutica indicada pelo profissional de saúde, complementaram o tratamento com chás e sucos, como indicam familiares e pessoas que passaram pela doença ou tiveram experiências com pessoas próximas.

O processo terapêutico dessas mulheres mostra, então, uma mescla do conhecimento profissional e das crenças populares, em que os tratamentos alternativos aparecem como uma ajuda na terapêutica tradicional. Esse processo é descrito como Sistemas de Cuidado à Saúde, que é constituído pela arena da cultura popular, pelos profissionais legalmente reconhecidos e pelos especialistas de cura não formais. ${ }^{22}$

O ser humano, desde o princípio de sua existência, tem buscado alternativas diversas na tentativa de eliminar seus males físicos e psíquicos. Então, o processo terapêutico dessas mulheres pode ser influenciado pelo contexto sóciocultural em que estão inseridas. ${ }^{23}$

Quando a vulnerabilidade é utilizada como marco conceitual em uma pesquisa, é preciso que se tome o cuidado de não transformá-la em uma reprodução do status quo pela naturalização da opressão, pois a pesquisa deve "produzir conhecimento para se emancipar as pessoas e grupo". ${ }^{24: 134}$ É imprescindível que sejam enfatizadas a resistência e a capacidade criadora e de superação dos indivíduos. ${ }^{24}$

Nesse sentido, pudemos apreender dos depoimentos das participantes deste estudo que diante da ameaça contra suas vidas, apegaram-se a tudo que pudesse, de alguma forma, por algum tempo, livrá-las da morte.

\section{CONSIDERAÇÕES FINAIS}

A vulnerabilidade das mulheres se mostrou tanto na possibilidade de adoecer, quando apresentavam algum sinal ou sintoma que as incomodava ou atrapalhava suas atividades de vida diária, quanto na realização do tratamento.

Encontramos as respostas para "como, porque e em que nível" a vulnerabilidade se apresenta a essas mulheres. Para as participantes deste estudo, essa apresentação aconteceu de forma crescente, a partir do aparecimento dos primeiros sinais e sintomas da doença, crescendo, à medida que o diagnóstico foi apresentado e o tratamento se fez necessário, trazendo dificuldades e sofri- mento, mas também, proporcionando a oportunidade dessas mulheres encontrarem formas de enfrentamento e superação, à medida do possível. Fatores relacionados à cliente, aos profissionais, aos serviços, entre outros, tornaram a mulher suscetível aos problemas e danos de sua saúde, relativos ao câncer cérvico-uterino, exacerbando sua vulnerabilidade à doença.

Os relatos evidenciando a vulnerabilidade ao câncer do colo do útero evidenciaram a necessidade de se superar algumas deficiências no modelo de assistência e na humanização do atendimento, no grau de compromisso e na qualidade das instituições, dos recursos, do gerenciamento e do monitoramento dos programas de prevenção e detecção do câncer do colo do útero, nos diferentes níveis de atenção.

\section{REFERÊNCIAS}

1. Ministério da Saúde (BR), Instituto Nacional do Câncer. Estimativa 2010: incidência de câncer no Brasil / Instituto Nacional do Câncer [online]. Rio de Janeiro (RJ): INCA; 2009 [acesso $2010 \mathrm{fev}$ 19]. Disponível em: http://www1.inca.gov.br/ estimativa/2010/

2. Organização Mundial da Saúde, Organização Pan-Americana da Saúde. $140^{a}$ sessão do Comitê Executivo. Estratégia e plano de ação regional para a prevenção e o controle de câncer do colo do útero. América Latina e Caribe, 2008-2015. Washington (US): OMS/OPAS; 2007.

3. Muller DK, Dias-da-Costa JS, Lus AMH, Olinto MTA. Cobertura do exame citopatológico do colo do útero na cidade de São Leopoldo, Rio Grande do Sul, Brasil. Cad Saúde Pública. 2008 Nov; 24(11):2511-20.

4. Lepique AP, Rabachini T, Villa LL. HPV vaccination: the beginning of the end of cervical cancer?: a review. Mem Inst Oswaldo Cruz. 2009 Fev; 104(1):1-10.

5. Nadal LRM, Nadal SR. Indicações da vacina contra o Papilomavirus Humano. Rev Bras Coloproctologia. 2008 Jan-Mar; 28(1):124-126.

6. Tucunduva LTCM, Sá VHLC, Koshimura ET, Prudente FVB, Santos AF, Samano EST, et al. Estudo da atitude e do conhecimento dos médicos não oncologistas em relação às medidas de prevenção e rastreamento do câncer. Rev Assoc Médica Brasileira. 2004 Jul-Set; 50(3):257-62.

7. Luciani S, Andrus JK. A Pan American Health Organization strategy for cervical cancer prevention and control in Latin America and the Caribbean. Reprod Health Matters. 2008 Nov; 16(32):59-66.

8. Otto SE. Neoplasias malignas ginecológicas. In: Otto SE. Oncologia. Rio de Janeiro (RJ): Reichmann \& Affonso Editores; 2002. p.160-82. 
9. Oliveira MMHN, Silva AAM, Brito LMO, Coimbra LC. Cobertura e fatores associados à não realização do exame preventivo de Papanicolaou em São Luís, Maranhão. Rev Bras Epidemio. 2006 Set; 9(3):325-34.

10. Amorim VMSL, Barros MBA, César CLG, Carandina L, Goldbaum M. Fatores associados à não realização do exame de Papanicolaou: um estudo de base populacional no município de Campinas, São Paulo, Brasil. Cad Saúde Pública. 2006 Nov; 22(11):2329-38.

11. Davim RMB, Torres GV, Silva RAR, Silva DAR. Conhecimento de mulheres de uma Unidade Básica de Saúde da cidade de Natal/RN sobre o exame de Papanicolaou. Rev Esc Enferm USP. 2005 Set; 39(3):296-302.

12. Aday La. At risk in América: the health and health care needs of vulnerable populations in the United States. San Franscico (US): Jossey-Bass; 1993.

13. Ayres JRCM, França Junior I, Calazans GJ, Saletti Filho HC. O conceito de vulnerabilidade e as práticas de saúde: novas perspectivas e desafios. In: Czeresnia D, Freitas CM. Promoção da saúde: conceitos, reflexões, tendências. Rio de Janeiro: Editora Fiocruz; 2003. p. 117-39.

14. Sanches AIM, Bertolozzi MR. Pode o conceito de vulnerabilidade apoiar a construção do conhecimento em saúde coletiva? Ciênc Saúde Coletiva. 2004 MaioJun; 12(2):319-34.

15. Paz AA, Santos BRL, Eidt OR. Vulnerabilidade e envelhecimento no contexto da saúde. Acta Paul Enferm. 2006 Jul-Set; 19(3):338-42.

16. Bardin L. Análise de conteúdo. $3^{\mathrm{a}}$ ed. Lisboa (PT): Edições 70; 2006.
17. Ministério da Saúde (BR), Conselho Nacional de Saúde, Comissão Nacional de Ética em Pesquisa. Resolução Nº 196 de 10 de outubro de 1996: diretrizes e normas regulamentadoras de pesquisa envolvendo seres humanos. Brasília (DF): MS; 1996.

18. Brenna SMF, Hardy E, Zeferino LC, Namura I. Conhecimento, atitude e prática do exame de Papanicolaou em mulheres com câncer de colo do uterino. Cad Saúde Pública. 2001 Jul-Ago; 17(4):909-14.

19. Thum M, Heck RM, Soares MC, Deprá AS. Câncer de colo uterino: percepção das mulheres sobre prevenção. Cienc Cuid Saude. 2008 Out-Dez; 7(4):509-16.

20. FernandesJV, RodriguesSHL, Costa YGA, Silva LCM, Brito AML, Azevedo JWV, et al. Conhecimentos, atitudes e prática do exame de Papanicolaou por mulheres, Nordeste do Brasil. Rev Saúde Pública. 2009 Out; 43(5):851-8.

21. Ministério da Saúde (BR). Departamento de Atenção Básica. Cadernos de Atenção Básica. Controle dos cânceres do colo do útero e de mama. Brasília (DF): MS; 2006.

22. Kleinman A. Patients and healers in the context of culture. California (US): Regents; 1980.

23. Siqueira KM, Barbosa MA, Brasil VV, Oliveira LMC, Andraus LMS. Crenças populares referentes à saúde: apropriação de saberes sócio-culturais. Texto Contexto Enferm. 2006 Jan-Mar; 15(1):68-73.

24. Nichiata LYI, Bertolozzi MR, Takahashi RF, Fracolli LA. A utilização do conceito "vulnerabilidade" pela enfermagem. Rev Latino-am Enfermagem. 2008 SetOut; 16(5):923-8. 